2. COVID-19 Dashboard by the Center for Systems Science and Engineering (CSSE) at Johns Hopkins University (JHU). Global Cases. Cited 30 Jul 2020. https://www.arcgis.com/apps/opsdashboard/index. html\#/bda7594740fd40299423467b48e9ecf6

3. Schulman S. Coronavirus disease 2019, prothrombotic factors, and venous thromboembolism. Semin Thromb Hemost 2020; 46: 772-6.

4. Thachil J, Srivastava A. SARS-2 coronavirus associated hemostatic lung abnormality in COVID-19: is it pulmonary thrombosis or pulmonary embolism? Semin Thromb Hemost 2020; 46: 777-80.

5. Levi M, Thachil J. Coronavirus disease 2019 coagulopathy: disseminated intravascular coagulation and thrombotic microangiopathy-either, neither, or both. Semin Thromb Hemost 2020; 46: 781-4.

6. Lippi G, Favaloro EJ D-dimer is associated with severity of coronavirus disease 2019: a pooled analysis. Thromb Haemost 2020; 120: 876-8.

7. Favaloro EJ, Lippi G. Recommendations for minimal laboratory testing panels in patients with COVID-19: potential for prognostic monitoring. Semin Thromb Hemost 2020; 46: 379-82.

8. Lippi G, Tripodi A, Simundic AM, Favaloro EJ. International survey on D-dimer test reporting: a call for standardization. Semin Thromb Hemost 2015; 41: 287-93.

9. Favaloro EJ, Thachil J. Reporting of D-dimer data in COVID-19: some confusion and potential for misinformation. Clin Chem Lab Med 2020 58: $1191-9$

10. Thachil C, Longstaff C, Favaloro EJ, Lippi G, Urano T, Kim PY. The need for accurate D-dimer reporting in COVID-19: Communication from the ISTH SSC on Fibrinolysis. J Thromb Haemost 2020; 18: 2408-11.

11. Standards for Pathology Informatics in Australia (SPIA). Reporting Terminology and Codes Haematology (v3.0). Sydney: RCPA, 2017. https://www.rcpa.edu.au/getattachment/bb8ce49b-26dd-490f-b544e35141028080/SPIA-Haematology-Reporting-Terminology-and-Codes. asp.

12. Longstaff C, Adcock D, Olson JD, et al. Harmonisation of D-dimer - a call for action. Thromb Res 2016; 137: 219-20.

DOI: https://doi.org/10.1016/j.pathol.2020.08.010

\section{Whole genome sequencing identifies opportunistic non- typeable Haemophilus influenzae rather than a hypervirulent clone}

Sir,

Haemophilus influenzae (HI) is a strict human pathogen implicated in respiratory tract infections. ${ }^{1}$ Invasive infections with capsulated $\mathrm{HI}$ type B (Hib) have all but disappeared due to Hib vaccination inclusion in the Australian immunisation program since $1993 .^{2}$ Occasionally, non-typeable HI (NTHI) are isolated from sterile sites, e.g., infective endocarditis, meningitis. ${ }^{3,4}$ The growing cohort of immunocompromised patients from malignancy, its treatment and organ transplantation create a susceptible population. ${ }^{5}$ In the absence of an opsonising and phagocytosis evading polysaccharide capsule, IgA proteases, serum resistance and adhesins are the main virulence characteristics for NTHI. ${ }^{1}$ We unexpectedly observed four cases of invasive NTHI over 7 months and investigated whether there was an unusually virulent clone circulating in our immunocompromised patient population.

Patients presented to our quaternary care metropolitan hospital from October 2018 to April 2019 with sterile site infections (Table 1). Bacterial identification was performed following isolation of pathogens using a matrix assisted laser desorption ionisation-time of flight mass spectrometry (MALDI-TOF MS; Bruker Daltonics, Germany) with HI identified by scores of $>2$. Susceptibility testing was performed according to Clinical and Laboratory Standards Institute (CLSI) M100 standards (28th ed) ${ }^{6}$ and $\beta$-lactamases detected by the nitrocefin disc test (Oxoid, UK).

Genomic DNA was extracted from the isolates using the Qiagen EZ1 Advanced Extractor (Qiagen, Germany) as per the manufacturer's instructions. Sequencing libraries were prepared using the Nextera DNA Flex Kit (Illumina, USA) as per the manufacturer's instructions and sequenced on an Illumina MiSeq. De novo assembly was performed on the read data using SKESA (v.2.3.0) and sequence typing performed using multilocus sequence typing (MLST). Virulence genes identified by Staples et al. were downloaded from NCBI and blasted against the assemblies. ${ }^{7}$ The sequencing reads were mapped to the complete genome Haemophilus influenzae strain NML-Hia-1 (GenBank accession: NZ_CP017811.1) using BWA and variants called using FreeBayes (v1.3.1-dirty). A maximum likelihood phylogenetic tree was constructed using FastTree (v2.1.10).

The clinical and microbiological characteristics of four male patients are shown in Table 1. Three patients were at risk for invasive NTHI infection from underlying defects in ear anatomy. The remaining patient was treated with rituximab for a lymphoproliferative disease. All were treated with ceftriaxone despite the availability of susceptibility testing. In the three bacteraemic patients, blood cultures were negative

Table 1 Clinical and microbiologic features in four patients with non-typeable Haemophilus influenzae invasive infection

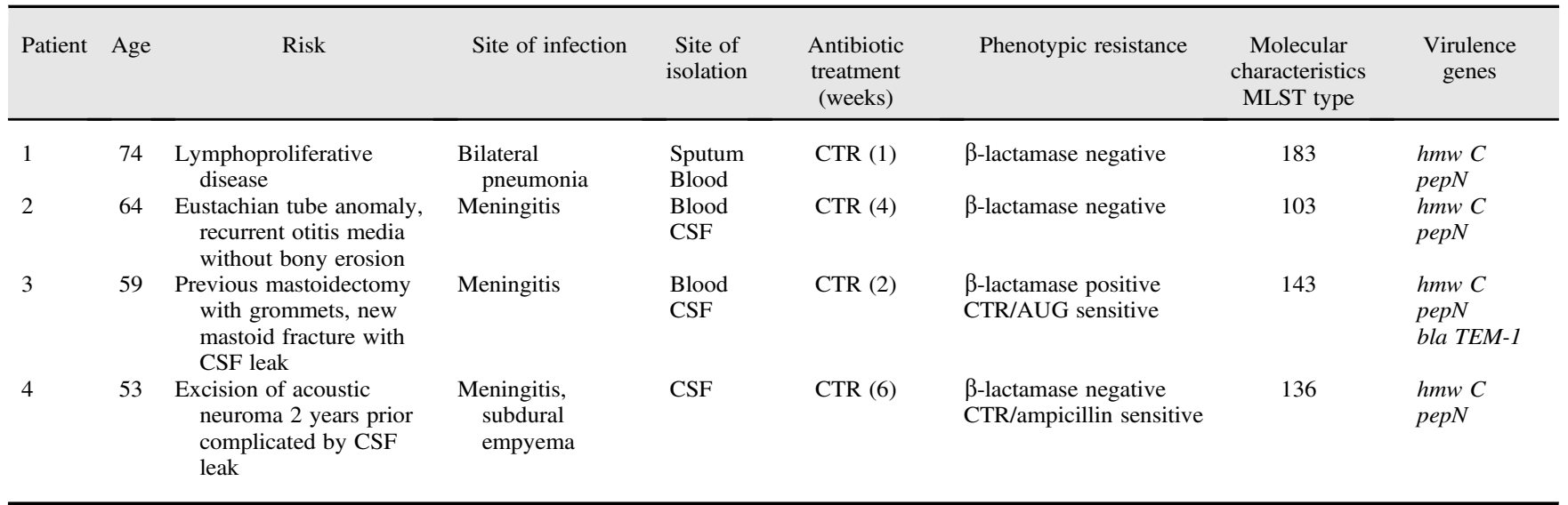


2-3 days after commencement of ceftriaxone. One patient, without bacteraemia, had NTHI isolated from cerebrospinal fluid (CSF). This patient had a poor outcome (hydrocephalus, intellectual deficits) despite negative CSF cultures $48 \mathrm{~h}$ postantibiotics. The four isolates were not identical based on in silico typing with more than 21,588 core SNPs between them (Fig. 1). Of the virulence genes investigated ( $h m w C$, pepN, $h m w 1 A, h m w 2 A$, hap, igAl), all four isolates harboured $h m w C$ and pepN. Absence of capsular BexA and Bex B genes confirmed the phenotypic absence of a capsule in all isolates. BlaTEM- 1 was detected in the single isolate testing $\beta$-lactamase positive using phenotypic methods.

Globally, the average incidence of invasive NTHI is approximately $1 / 100000$ population. ${ }^{8}$ Reported reasons for the rise in NTHI infections include increases in nasopharyngeal colonisation in the healthy population from strain replacement post-Hib vaccination. This results in increased transmission of NTHI to vulnerable individuals. Typically, patients infected with NTHI have advancing age and underlying comorbidity, e.g., cardiopulmonary disease, HIV, alcoholism, malignancy, splenectomy, hypogammaglobulinaemia, etc. ${ }^{1,8}$ Our findings were consistent with the literature where one patient had lymphoma, while the remaining patients had meningitis related to anatomical defects and prior surgery. Although invasive infection is rare and our cases occurred over a short 7 month period, genome sequencing did not reveal clonal spread or novel virulence factors. Staples et al. also reported high genetic diversity by MLST and SNP typing without a dominant clone in 74 NTHI strains over a 15-year period in Queensland. ${ }^{7}$ However, Andersson et al. reported a respiratory outbreak in a Swedish long term care facility where all isolates clustered as MLST 14. ${ }^{9}$ Virulence factors associated with invasive NTHI are less well described. Biofilms prevent clearance in children with otitis media. ${ }^{10}$ Two Ig A proteases (igaA, igaB genes) are

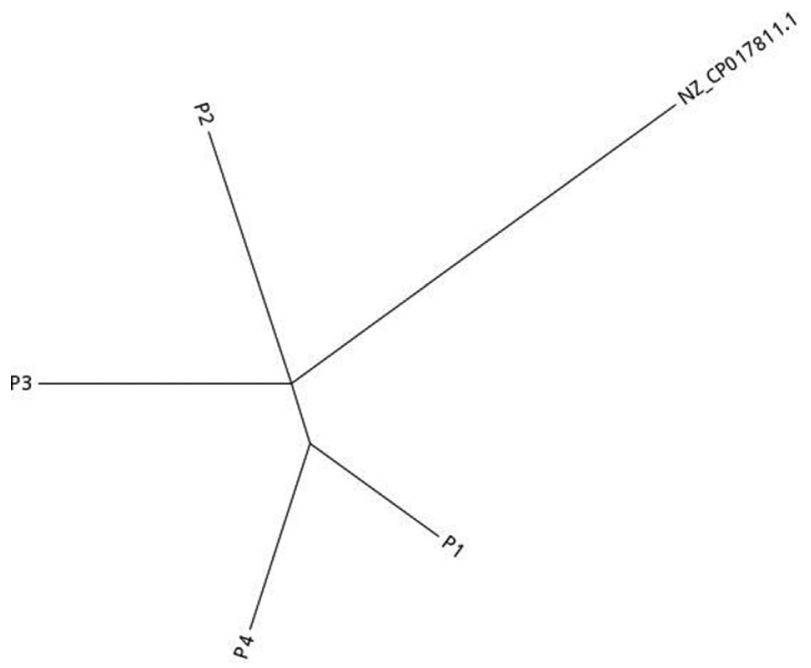

$\overline{0.07}$

Fig. 1 Maximum likelihood phylogeny depicting the genetic relationship among isolates using Haemophilus influenzae strain NML-Hia-1 (GenBank accession: NZ_CP017811.1) as a reference. Scale bar indicates nucleotide substitutions per site. implicated in chronic obstructive pulmonary disease associated pneumonia; serum resistance in infective endocarditis and adhesin proteins are described. ${ }^{5,11}$ Sequencing detected the presence of $h m w C$ and pepN adhesins in our isolates. Adhesins mediate the attachment to human epithelial cells and promote colonisation. It is likely that our patients with meningitis were colonised with NTHI, and contiguous meningeal spread facilitated by disruption of anatomical planes was the pathogenesis.

In conclusion, the availability of whole genome sequencing in a clinical laboratory rapidly addressed our concerns of an unusually virulent clone or possible outbreak. NTHI have been implicated in clonal invasive disease, hence continuous surveillance and application of rapid genomic methods is relevant, particularly in our severely immunocompromised population.

Acknowledgements: We thank the laboratory scientific staff at Royal Prince Alfred Hospital.

Conflicts of interest and sources of funding: The authors state that there are no conflicts of interest or funding sources to disclose.

\section{Melanie-Anne John, Alicia Beukers, Raymond Chan, Sebastiaan van Hal}

Department of Microbiology and Infectious Diseases, New South Wales Health Pathology, Royal Prince Alfred Hospital, Sydney, NSW, Australia

Contact Dr Melanie-Anne John.

E-mail: melanieanne.john@health.nsw.gov.au

1. Murphy TF. Haemophilus species (including chancroid). In Mandell GL, Bennett JE, Dolin R, editors. Principles and Practice of Infectious Disease. 7th ed. Philadelphia, PA: Elsevier, 2010; 2911-9.

2. Horby P, Gilmour R, Wang H, McIntyre P. Progress towards eliminating Hib in Australia: an evaluation of Haemophilus influenzae type b prevention in Australia, 1 July 1993 to 30 June 2000. Commun Dis Intell 2003; 27: 324-41.

3. Darras-Joly C, Lortholary O, Mainardi JL, et al. Haemophilus endocarditis: report of 42 cases in adults and review. Clin Infect Dis 1997; 24: 1087-94.

4. Dworkin MS, Park L, Borchardt SM. The changing epidemiology of invasive Haemophilus influenzae disease, especially in persons $\geq 65$ years old. Clin Infect Dis 2007; 44: 810-6.

5. Bertelle-Ibrahim LA, Murphy TF, Kirkham C, et al. Non-typeable Haemophilus influenzae infective endocarditis in a renal transplant recipient: compromised host or virulent strain? BMJ Case Rep 2013; 2013: bcr2013200377.

6. Clinical and Laboratory Standards Institute (CLSI). M100: Performance Standards for Antimicrobial Susceptibility Testing. $28^{\text {th }}$ ed. Wayne, PA: CLSI, 2018.

7. Staples M, Graham RMA, Jennison AV. Characterisation of invasive Haemophilus influenza isolates in Queensland, Australia using whole genome sequencing. Epidemiol Infect 2017; 145: 1727-36.

8. Langerereis JD, de Jonge MI. Invasive disease caused by nontypeable haemophilus influenzae. Emerg Infect Dis 2015; 21: 1711-8.

9. Andersson M, Resman F, Eitrem R, et al. Outbreak of a beta-lactam resistant non-typeable Haemophilus influenzae sequence type 14 associated with severe clinical outcomes. BMC Infect Dis 2015; 15: 581-7.

10. Murphy TF, Faden H, Bakaletz LO, et al. Nontypeable Haemophilus influenzae as a pathogen in children. Pediatr Infect Dis 2009; 28: 43-8.

11. Murphy TF, Lesse AJ, Kirkham C, et al. A clonal group of nontypeable Haemophilus influenzae with two IgA proteases is adapted to infection in chronic obstructive pulmonary disease. PLoS One 2011; 6: e25923.

DOI: https://doi.org/10.1016/j.pathol.2020.08.011 